were there any differences in blood cell count, the authors acknowledge the need to further assess any adverse effects of bortezomib in these mice. Another limitation is that only one cycle of doses was administered. Nevertheless, the data indicate a therapeutic potential for bortezomib in rheumatoid arthritis.

Original article Lee SW et al. (2008) Bortezomib attenuates murine collagen-induced arthritis. Ann Rheum Dis [doi:10.1136/ard.2008.097709]

\section{Respiratory ciliary function is impaired in Wegener's granulomatosis}

A new study has suggested that impaired mucociliary function has a role in the pathogenesis of Wegener's granulomatosis (WG). Ullrich et al. chose to investigate the ciliary beat frequency in WG, as the disease typically begins with granulomatous inflammation of the respiratory tract. Previous research has proposed that reduced ciliary function might be a result of, or a causal factor leading to, inflammation and an increased risk of infection of the respiratory tract.

Ciliary beat frequency was analyzed in 30 patients with WG (involving the upper respiratory tract), 12 patients with other rheumatic disease (disease controls), and 10 healthy controls. Nasal epithelial cells were obtained from all participants, and measurements of ciliary beat frequency were made after $5 \mathrm{~h}$ and $24 \mathrm{~h}$.

The ciliary beat frequency values in the healthy control group were within the predetermined normal range at $5 \mathrm{~h}$ and $24 \mathrm{~h}$, whereas the corresponding values were notably lower in patients with WG and disease controls at both time points. Compared with the disease controls, patients with WG had a greater reduction in ciliary beat frequency after $24 \mathrm{~h}$-indeed, ciliary function was almost idle in the latter. A correlation between the cumulative number of immunosuppressant agents and impairment of ciliary function was observed in patients with WG.

Ullrich et al. recommend a larger cohort study to confirm these data; however, in light of these initial results, they suggest that patients with
WG avoid any activities that might impair ciliary function, such as taking nasal decongestants or smoking.

Original article Ullrich S et al. (2008) Severely impaired respiratory ciliar function in Wegener's granulomatosis. Ann Rheum Dis [doi:10.1136/ard.2008.096974]

\section{Cardiotrophin-1 signaling essential for normal mouse bone resorption and formation}

Cardiotrophin-1 (CT-1) signaling has a fundamental role in a variety of biological systems, but has yet to be studied in bone. Walker et al. present the first evidence suggesting that CT-1 signaling is crucial for normal bone metabolism in mice.

Strong CT-1 expression in the osteoclasts, but not osteoblasts, of neonatal mouse bone samples prompted the authors to examine the effects of CT-1 in vitro and in vivo. They observed a dose-dependent, CT-1-induced increase in bone mineralization and osteoblast activity in a mouse stromal Kusa 4b10 cell line, which could indicate a novel role for CT-1 as a 'coupling factor' in bone formation. In addition, CT-1 administration increased the bone formation rate in wild-type mice, possibly via a CT1 -induced CAAT/enhancer-binding protein- $\delta$ signaling pathway, although the osteoblast mineralizing surface over which bone is formed was not altered.

Bone samples of CT-1 knockout mice revealed osteopenia and impaired mineralization, as well as more osteoclasts and fewer osteoblasts than in wild-type mice. CT-1 knockout mice also showed impaired bone resorption in vivo, but not in vitro, suggesting that defective bone resorption is a non-cellautonomous trait. Interestingly, the osteoblast surface was normal in adult mice, which, together with only a mild and transient reduction in bone formation, resulted in the reversal of osteopenia with age. Impaired bone resorption, however, was retained, and led to the development of osteopetrosis.

\footnotetext{
Original article Walker EC et al. (2008) Cardiotrophin-1 is an osteoclast-derived stimulus of bone formation required for normal bone remodelling. J Bone Miner Res 23: 2025-2032
} 